\title{
Calculation of Exciton and Trion Absorption Spectra in High Magnetic Fields
}

\author{
A. Geadysiewicz and A. Wójs \\ Institute of Physics, Wrocław University of Technology \\ Wybrzeże Wyspiańskiego 27, 50-370 Wrocław, Poland
}

\begin{abstract}
Density of states and absorption spectrum of narrow quantum wells containing a small number of free electrons and subject to a high magnetic field are calculated numerically. The effect of an additional, second electron on the photoexcited electron-hole pair is analyzed. In density of states, the exciton-electron interaction fills the gaps between the Landau levels and yields additional discrete peaks corresponding to bound trions. In absorption, interaction with the additional free electron has no effect on the position or intensity of the main sequence of excitonic peaks. However, it gives rise to additional weaker trion peaks, both in the lowest and higher Landau levels.
\end{abstract}

PACS numbers: 71.35.Pq, 71.35.Ji, 71.10.Pm

\section{Introduction}

The exciton is an electron-hole pair bound by the Coulomb attraction, $\mathrm{X}=\mathrm{e}+\mathrm{h}$. It occurs naturally in photoluminescence (PL) experiments, since the relaxation time of both photoexcited carriers is typically much shorter than the radiative recombination time. Negative and positive trions consist of an exciton and an additional bound electron or hole, $\mathrm{X}^{-}=\mathrm{X}+\mathrm{e}$ or $\mathrm{X}^{+}=\mathrm{X}+\mathrm{h}$, respectively. Whether trions actually form from excitons depends on the presence of free carriers in the system (e.g., due to doping), in addition to photoexcited pairs. The measure of the attraction between a neutral exciton and the free carrier is called the binding energy of a given trion state. It is defined as a difference between the ground state energy of the unbound configuration (an exciton and an electron in their separate ground states) and the trion energy. Obviously, trion binding energies (originating from charge-dipole interaction) are much smaller than exciton binding energy (due to direct charge-charge attraction). However, the spatial confinement and the magnetic field can profoundly increase the exciton-electron attraction [1], allowing for observation of these complexes in the experimental PL spectra [2]. 
Trions in 2D quantum wells (QWs) have been the subject of extensive theoretical studies in the past [3-5]. Depending on such parameter as the well width $w$ or magnetic field strength $B$, their energy spectrum contains one or more bound states. They are labeled by spin $S$ of the two identical carriers and total relative angular momentum $M$. Due to a simple optical selection rule, only those trions with $M=0$ can recombine without transferring their angular momentum to other carriers or impurities (which may be sparse in high-quality wells at low carrier concentration). Hence, the $M=0$ trions are called "bright", in contrast to the $M \neq 0$ "dark" states.

At least three trions are identified in model calculations: "bright singlet" $\mathrm{X}_{\mathrm{s}}^{-}(S=0, M=0)$ which is the ground state at small $B[1]$, "dark triplet" $\mathrm{X}_{\mathrm{td}}^{-}$ $(S=1, M=-1)$ predicted to survive as the only bound state in the high- $B$ limit [3], and a weakly bound but strongly radiative "bright triplet" $\mathrm{X}_{\mathrm{tb}}^{-}(S=1$, $M=0$ ) that occurs at intermediate fields [5]. All or some of these states are usually observed in PL experiments [6-8].

Understanding the role of trions in PL experiments requires many factors to be taken into account (e.g., complicated single-particle energy band structure, inter-carrier interactions, lattice defects, or spin-related effects). Some of the experimentally measured parameters can be calculated rather accurately, e.g. binding energies [5]. In this paper we discuss the effect of the presence of a trion on the optical absorption spectra of two-dimensional electron gas (2DEG) in high magnetic fields. The attention is paid mainly to the interaction of exciton with single free electron and its effect on the PL spectrum.

\section{Model}

We consider a symmetric GaAs quantum well of width $w=15 \mathrm{~nm}$, weakly doped to produce a small electron concentration $\rho$, and placed in a high magnetic field $B=10 \mathrm{~T}$. By small concentration we mean that the filling factor $\nu=2 \pi \rho \lambda^{2}$ is much smaller than unity $(\lambda=\sqrt{h c / e B}$ being the magnetic length). The $\mathrm{e}+\mathrm{h}$ and $2 \mathrm{e}+\mathrm{h}$ energy spectra were obtained by exact numerical diagonalization of the Hamiltonian matrix. All calculations were performed on the Haldane sphere [9]. In this geometry, the magnetic field normal to the spherical surface of radius $R$ is produced by a Dirac monopole $2 Q=4 \pi R^{2} / \phi_{0}$ (where $\phi_{0}=h c / e$ is the magnetic flux quantum) placed at its center. The single-particle energy levels $\varepsilon_{n}$ fall into degenerate angular momentum shells with $l=Q+n$. The $n$-th shell represents the $n$-th Landau level (LL), and $n=0$ corresponds to the lowest LL. The energy $\varepsilon_{n}$ consists of the cyclotron energy $\hbar \omega_{\mathrm{c}}\left(n+\frac{1}{2}\right)$ and a curvature-dependent finite-size term $n(n+1) \hbar^{2} / 2 \mu R^{2}$. In the above, $\omega_{\mathrm{c}}=e B / \mu c$ is the cyclotron frequency, and $\mu$ is the electron or hole effective mass.

With the aim of realistic modeling we replaced $\varepsilon_{n}$ with the correct energies of electron and hole LLs, taken from experiment, i.e. at $B=10 \mathrm{~T}, \hbar \omega_{\mathrm{c}}=17.8 \mathrm{meV}$ for electrons and $3.7 \mathrm{meV}$ for the heavy hole [10]. The Coulomb interaction matrix 
elements were integrated numerically in 3D using the lowest-subband electron and hole wave functions $\phi(z)$.

\section{Density of states}

First, we have calculated the density of states DOS $=\mathrm{d} \Gamma / \mathrm{d} E$ (the number of states $\Gamma$ per unit of energy $E$ ). Figure 1 shows results for $\mathrm{e}+\mathrm{h}$ and $2 \mathrm{e}+\mathrm{h}$, both for large $2 Q=50$ and with only two lowest electron and hole LLs included $(n \leq 1)$. Discrete energy spectra were transformed into continuous lines by broadening with Gaussian functions. Solid, dashed and dotted lines were used to mark $2 \mathrm{e}+\mathrm{h}$ ( $S=0$ and 1 plotted separately) and e $+\mathrm{h}$ curves, respectively. Only $S_{z}=0$ is shown. Additionally, the $2 \mathrm{e}+\mathrm{h}$ curve was rescaled so that comparison with $\mathrm{e}+\mathrm{h}$ lines can be drawn.

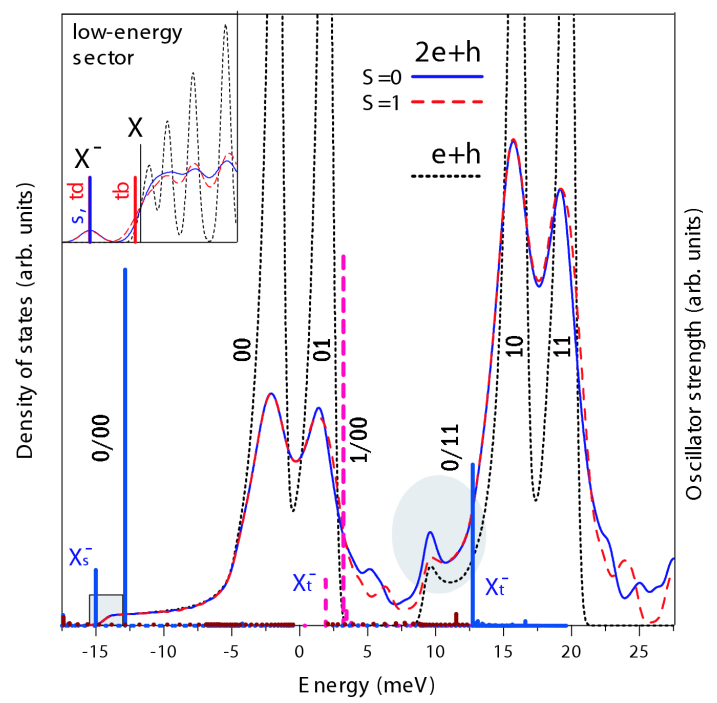

Fig. 1. Density of states (DOS) of $\mathrm{e}+\mathrm{h}$ and $2 \mathrm{e}+\mathrm{h}$ systems (continuous lines) and optical DOS for $2 \mathrm{e}+\mathrm{h}$ (discrete bars), calculated for a symmetric $15 \mathrm{~nm}$ GaAs quantum well at magnetic field $B=10 \mathrm{~T}$. Inset: magnified lowest-energy sector.

One of the differences between $2 \mathrm{e}+\mathrm{h}$ and $\mathrm{e}+\mathrm{h}$ DOS is the emergence of trion states below excitonic continuum. For the noninteracting e-h pairs DOS contains discrete peaks labeled by " $n_{\mathrm{e}} n_{\mathrm{h}}$ ". When interactions are included, these peaks broaden and shift slightly to the lower energies.

The curves for $2 \mathrm{e}+\mathrm{h}$ are dominated by " $n_{\mathrm{e}} n_{\mathrm{e}}^{\prime} n_{\mathrm{h}}$ " peaks, corresponding to three unbound particles confined in different combinations of LLs. Low-energy tails are due to exciton plus an additional unbound electron. Bound trions states are visible as discrete peaks just below continuum (see inset for clarity). They are the $\mathrm{X}_{\mathrm{s}}, \mathrm{X}_{\mathrm{td}}, \mathrm{X}_{\mathrm{tb}}$ (the weak binding of $\mathrm{X}_{\mathrm{s}}$ is a result of unrealistic restriction to $n \leq 1)$. 


\section{Optical DOS and absorption spectra}

For a pair of initial and final states $|\mathrm{i}\rangle=\mathrm{e}$ and $|\mathrm{f}\rangle=2 \mathrm{e}+\mathrm{h}$, the optical oscillator strength is $\Omega_{\mathrm{if}}=|\langle\mathrm{f}|\mathcal{P}| \mathrm{i}\rangle|^{2}$, with $\mathcal{P}$ defining the dipole transition. It can be expressed as a function of either initial- or final-state energy, and e.g., $\Omega(E)=\sum_{\mathrm{if}} \Omega_{\mathrm{if}} \delta\left(E_{\mathrm{f}}-E\right)$ describes the optical DOS (ODOS) of the $2 \mathrm{e}+\mathrm{h}$ system. Analogous expressions hold for the vac $\leftrightarrow \mathrm{e}+\mathrm{h}$ transition.

On the other hand, the oscillator strength may be expressed as a function of the photon energy $\mathcal{E}=E_{\mathrm{f}}-E_{\mathrm{i}}$ and weighted by the initial-state occupation function $\Theta_{\mathrm{i}}$, to give the absorption spectrum $\Omega(\mathcal{E})=\sum_{\mathrm{if}} \Omega_{\mathrm{if}} \Theta_{\mathrm{i}} \delta\left(E_{\mathrm{f}}-E_{\mathrm{i}}-\mathcal{E}\right)$. Let us note that this is obviously not equivalent ODOS for $2 \mathrm{e}+\mathrm{h}$.

Figure 2 plots ODOS of $\mathrm{e}+\mathrm{h}$, for $n \leq 4$ and $2 Q=20$. Empty dashed bars correspond to a noninteracting $\mathrm{e}-\mathrm{h}$ pair. They have equal height and are equally spaced by the joint electron and hole cyclotron gap $\hbar \omega_{\mathrm{c}, \mathrm{X}} \equiv \hbar\left(\omega_{\mathrm{c}, \mathrm{e}}+\omega_{\mathrm{c}, \mathrm{h}}\right)$. Empty solid bars illustrate the situation in which the $\mathrm{e}-\mathrm{h}$ interaction is so weak compared to $\hbar \omega_{\mathrm{c}, \mathrm{X}}$ that the LL mixing is absent. The peaks still have equal heights but now their positions shift by $\Delta_{\mathrm{X}}(n)$, the excitonic binding energy in the $n$-th LL. Since $\Delta_{\mathrm{X}}(n)$ decreases with $n$, the separation between consecutive peaks also decreases (and is always larger than $\hbar \omega_{\mathrm{c}, \mathrm{X}}$ ). Finally, the grey-filled bars show the spectrum including both intra- and inter-LL interaction. The strongest effect of the LL mixing is the intensity transfer from higher to lower energy.

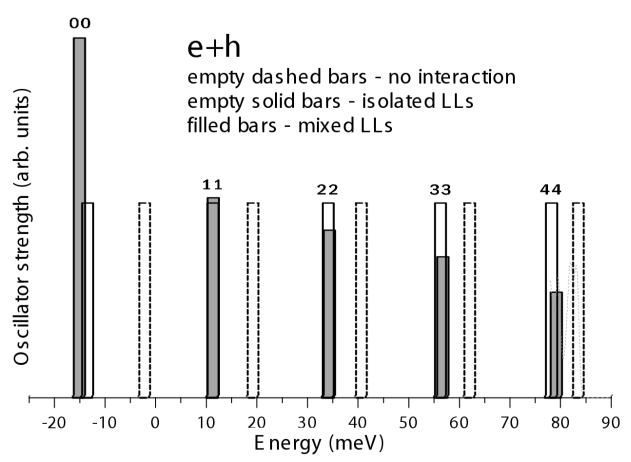

Fig. 2. ODOS of e+h, in a $15 \mathrm{~nm}$ symmetric GaAs QW at the magnetic field $B=10 \mathrm{~T}$. Five lowest LLs were included in the calculation.

Figure 1 shows also optical DOS for $2 \mathrm{e}+\mathrm{h}$, calculated using the same $n \leq 1$ and $2 Q=50$. There is no apparent correlation between DOS and optical DOS. In analogy to e $+\mathrm{h}$, the main peaks, labeled by " $n^{\prime} / n n$ ", correspond to a zero-wave vector exciton created/annihilated on the $n$-th LL, in the presence of the second electron (not bound to the exciton) on the $n^{\prime}$-th LL. These peaks describe optical processes almost unaffected by exciton-electron scattering. Optically active bound trions (with e and h residing in different LLs) are visible as weaker peaks displaced from " $n / n n$ " by the appropriate binding energy. In the lowest LL, only the singlet 
state is well visible because $\mathrm{X}_{\mathrm{td}}^{-}$has no oscillator strength, and $\mathrm{X}_{\mathrm{tb}}$ has very small binding energy (so that it cannot be distinguished from the exciton). The triplet trion visible at $E \approx 2.5 \mathrm{meV}$ has one of its electrons in the 1st excited LL. It can be formed if " 00 " exciton captures thermally excited electron.

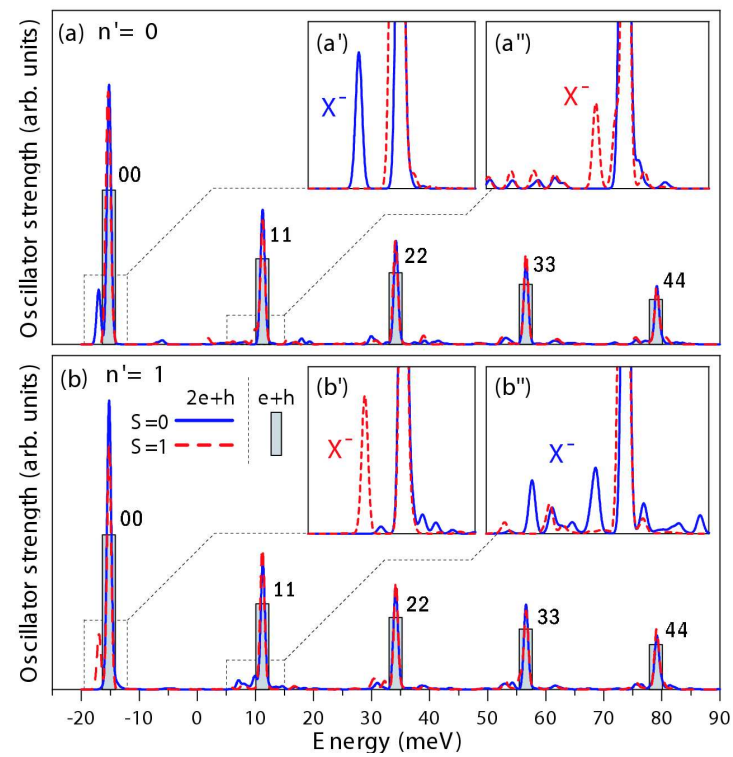

Fig. 3. The spin-resolved $2 \mathrm{e}+\mathrm{h}$ absorption spectra calculated for a symmetric $15 \mathrm{~nm}$ GaAs quantum well at magnetic field $B=10 \mathrm{~T}$ including five lowest LLs. Parts (a) and (b) correspond to the initial-state electron in the lowest or first excited LL. Insets show the magnified regions around the lowest two peaks.

Figure 3 shows the $2 \mathrm{e}+\mathrm{h}$ absorption spectra $\Omega(\mathcal{E})$, assuming that the initial-state electron is either in the lowest or first excited LL. Remarkably, when the (great number of) main ODOS peaks " $n^{\prime} / n n^{\prime}$ " are shifted by $E_{\mathrm{i}}=n^{\prime} \hbar \omega_{\mathrm{c}, \mathrm{e}}$, they reproduce the bare-exciton spectrum.

The presence of an additional electron does not cause shifting or splitting of these main absorption energies, and it has no significant effect on their intensities (relative to one another). This demonstrates that bare excitonic absorption is unaffected by a dilute electron gas (neither by renormalization of energy nor by transfer of intensity between LLs).

The effect of free electrons is emergence of additional (compared to the bare exciton) features in absorption spectrum, the strongest of them associated with the trion formation. In Fig. 3, trion absorption peaks can be seen most clearly in the insets, in which the vicinities of peaks " 00 " and " 11 " have been magnified. In reality, their intensity relative to the excitonic peaks will depend on the filling factor $\nu$ (and can easily be much higher). 


\section{Summary}

In conclusion, we have calculated the DOS for $\mathrm{e}+\mathrm{h}$ and $2 \mathrm{e}+\mathrm{h}$ systems by exact numerical diagonalization of the realistic Hamiltonians. The main difference between DOS of $\mathrm{e}+\mathrm{h}$ and $2 \mathrm{e}+\mathrm{h}$ is the emergence of discrete bound trion states below the excitonic tails and redistribution of DOS away from the LL peaks. The ODOS of $2 \mathrm{e}+\mathrm{h}$ shows no obvious correlation with DOS. The presence of (and interaction with) an additional electron manifests in form of additional weaker peaks. Some of them are attributed to bound trion states (in the lowest and higher LLs).

\section{Acknowledgments}

The authors gratefully acknowledge discussions with J.J. Quinn and L. Bryja. Work supported by grant No. 1P03B03230 of the MENiS (Poland).

\section{References}

[1] B. Stebe, A. Ainane, Superlatt. Microstruct. 5, 545 (1989).

[2] K. Kheng, R.T. Cox, Y. Merle d'Aubigné, F. Bassani, K. Saminadayar, S. Tatarenko, Phys. Rev. Lett. 71, 1752 (1993); H. Buhmann, L. Mansouri, J. Wang, P.H. Beton, N. Mori, M. Heini, M. Potemski, Phys. Rev. B 51, 7969 (1995); G. Finkelstein, H. Shtrikman, I. Bar-Joseph, Phys. Rev. Lett. 74, 976 (1995); Phys. Rev. B 53, R1709 (1996); A.J. Shields, M. Pepper, M.Y. Simmons, D.A. Ritchie, Phys. Rev. B 52, 7841 (1995).

[3] A. Wójs, P. Hawrylak, Phys. Rev. B 51, 10880 (1995); J.J. Palacios, D. Yoshioka, A.H. MacDonald, Phys. Rev. B 54, 2296 (1996); A.B. Dzyubenko, A.Y. Sivachenko, Phys. Rev. Lett. 84, 4429 (2000).

[4] D.M. Whittaker, A.J. Shields, Phys. Rev. B 56, 15185 (1997); C. Riva, F.M. Peeters, K. Varga, ibid. 61, 13873 (2000); ibid. 63, 115302 (2001).

[5] A. Wójs, J.J. Quinn, P. Hawrylak, Phys. Rev. B 62, 4630 (2000).

[6] M. Hayne, C.L. Jones, R. Bogaerts, C. Riva, A. Usher, F.M. Peeters, F. Herlach, V.V. Moshchalkov, M. Henini, Phys. Rev. B 59, 2927 (1999).

[7] G. Yusa, H. Shtrikman, I. Bar-Joseph, Phys. Rev. Lett. 87, 216402 (2001).

[8] T. Vanhoucke, M. Hayne, M. Henini, V.V. Moshchalkov, Phys. Rev. B 63, 125331 (2001); ibid. 65, 041307 (2002); G.V. Astakhov, D.R. Yakovlev, V.V. Rudenkov, P.C.M. Christianen, T. Barrick, S.A. Crooker, A.B. Dzyubenko, W. Ossau, J.C. Maan, G. Karczewski, T. Wojtowicz, Phys. Rev. B 71, 201312 (2005); D. Andronikov, V. Kochereshko, A. Platonov, T. Barrick, S.A. Crooker, G. Karczewski, Phys. Rev. B 72, 165339 (2005).

[9] F.D.M. Haldane, Phys. Rev. Lett. 51, 605 (1983).

[10] B.E. Cole, J.M. Chamberlain, M. Henini, T. Cheng, W. Batty, A. Wittlin, J.A.A.J. Perenboom, A. Ardavan, A. Polisski, J. Singleton, Phys. Rev. B 55, 2503 (1997). 